\title{
Oxidative para-Triflation of Acetanilides
}

\author{
Amélie Pialat, Benoît Liégault, ${ }^{\star}$ and Marc Taillefer* \\ Institut Charles Gerhardt, UMR-CNRS 5253, AM2N \\ ENSCM, 8 rue de l'école normale, 34296 Montpellier Cedex 5, France \\ benoit.liegault@enscm.fr
}

\section{Table of Contents}

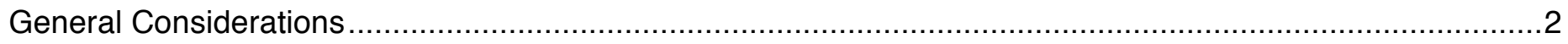

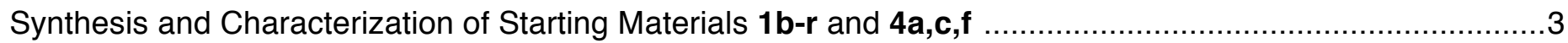

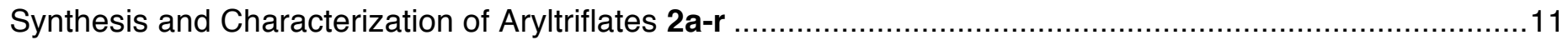

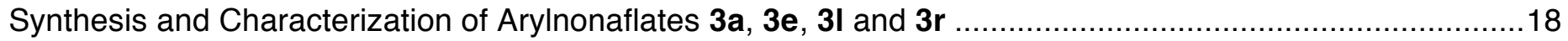

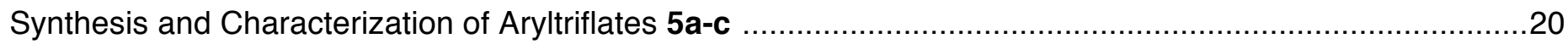

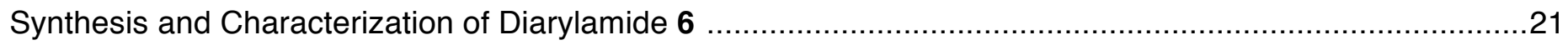

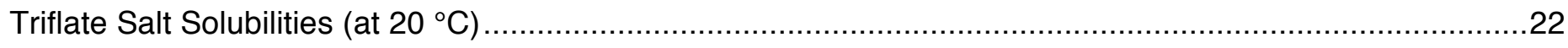




\section{General Considerations}

$\mathrm{Phl}(\mathrm{OAc})_{2}, \mathrm{Phl}\left(\mathrm{OCOCF}_{3}\right)_{2}$ and AgOTf were purchased from Sigma-Aldrich and Strem Chemicals, respectively, stored in a dessicator cabinet and weighed to air. AgONf was prepared according to a literature procedure, ${ }^{1}$ stored in a dessicator cabinet and weighed to air. Anilines, anilides $\mathbf{1 a}$ and $\mathbf{4 b}$ and other reagents were purchased from SigmaAldrich, Alfa Aesar, Acros or TCl, and used as received. High-quality grade $\mathrm{CHCl}_{3}, \mathrm{DCE}, \mathrm{CH}_{2} \mathrm{Cl}_{2}$ and THF were purified/dried on an MBraun Solvent Purification System and stored in Schlenk flasks under argon over $3 \AA$ molecular sieves. Analytical thin layer chromatographies (TLC) were carried out using Merck silica gel $60 \mathrm{~F}_{254}$ plates and visualized under UV light $(254 \mathrm{~nm})$. Starting materials were purified by manual flash column chromatography with Merck Geduran Si 60 silica gel $(40-63 \mu \mathrm{m})$. Aryltriflates and arylnonaflates were purified by automatic flash column chromatography (on an Armen Instrument Spot) with $25 \mathrm{~g}$ PF-30SiHP Interchim pre-packed silica gel columns.

Triflation and nonaflation reactions were performed on $0.25-0.5 \mathrm{mmol}$ scale in oven-dried $4 \mathrm{~mL}$ screw-cap vials under argon and analyzed by GC-MS (on a Shimadzu QP-2010SE) and ${ }^{19} \mathrm{~F}$ NMR in $\mathrm{CD}_{3} \mathrm{OD}$ using 4,4'-difluorobenzophenone as an internal standard (relaxation time set at $10 \mathrm{~s}$ ).

${ }^{1} \mathrm{H},{ }^{13} \mathrm{C}$ and ${ }^{19} \mathrm{~F}$ NMR spectra were recorded at 298K with Bruker AC-400 or DRX-250 spectrometers and referenced from TMS (in the case of ${ }^{1} \mathrm{H}$ NMR spectra run in $\mathrm{CDCl}_{3}$ ) or from the residual solvent signal (in other cases). Data are reported as follows: chemical shift (in ppm) [multiplicity $(s=$ singlet, $d=$ doublet, $t=$ triplet, $q=$ quadruplet, $m=$ multiplet, $\mathrm{br}=$ broad), coupling constant (in $\mathrm{Hz}$ ), integration)]. HRMS (Q-TOF) were performed on a JEOL JMS-DX300 spectrometer ( $3 \mathrm{keV}$, xenon) in a $m$-nitrobenzylalcohol matrix. Melting points (when applicable) were carried out on a Büchi B-540 melting point apparatus.

Copies of ${ }^{1} \mathrm{H},{ }^{13} \mathrm{C}$ and ${ }^{19} \mathrm{~F}$ NMR spectra for aryltriflates and arylnonaflates can be found in a separate file. 


\section{Synthesis and Characterization of Starting Materials $1 b-r$ and $4 a, c, f$}

\section{General Procedure A}

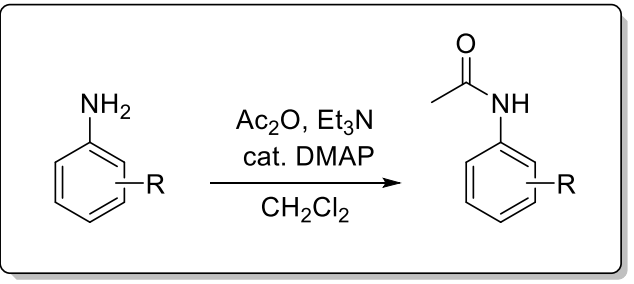

To a solution of the desired aniline (10-15 mmol scale, 1.0 equiv) and triethylamine (2.2 equiv) in $\mathrm{CH}_{2} \mathrm{Cl}_{2}(0.4 \mathrm{M})$ at $0{ }^{\circ} \mathrm{C}$ under argon was added acetic anhydride (1.2 equiv) dropwise. The resulting mixture was warmed to room temperature and stirred until the reaction was judged to be complete by TLC (2-16 hours). Water was added and the crude product was extracted with $\mathrm{CH}_{2} \mathrm{Cl}_{2}$. The combined organic phases were washed with saturated solutions of $\mathrm{NaHCO}_{3}$ and $\mathrm{NH}_{4} \mathrm{Cl}$, dried over $\mathrm{MgSO}_{4}$ and concentrated under reduced pressure. The product was purified by silica gel flash chromatography.

\section{General Procedure $B^{2}$}

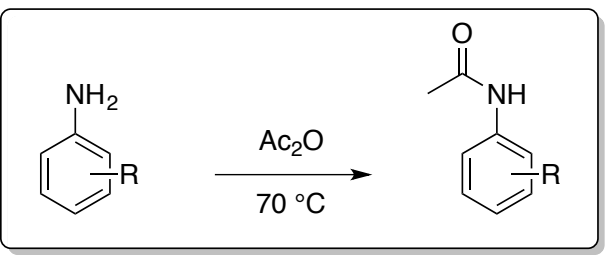

To a solution of acetic anhydride (2.0 equiv) at $0^{\circ} \mathrm{C}$ under argon was added the desired aniline (10-15 mmol scale, 1.0 equiv). The resulting mixture was stirred at $70^{\circ} \mathrm{C}$ until the reaction was judged to be complete by TLC (2-16 hours). The excess of acetic anhydride was evaporated under reduced pressure and the product was purified by silica gel flash chromatography.<smiles>CC(=O)Nc1ccccc1Br</smiles>

\section{2'-Bromoacetanilide (1b)}

Prepared according to general procedure B, from 2-bromoaniline (36\%).

Exhibited spectral data identical to a previous report. ${ }^{3}$

$\mathbf{R}_{\mathrm{f}} 0.4$ (petroleum ether/EtOAc 70:30)

${ }^{1} \mathrm{H}$ NMR $\left(400 \mathrm{MHz}, \mathrm{CDCl}_{3}, 298 \mathrm{~K}\right) \delta 8.31(\mathrm{~d}, J=8.0 \mathrm{~Hz}, 1 \mathrm{H}), 7.62(\mathrm{br} \mathrm{s}, \mathrm{NH}), 7.52(\mathrm{dd}, J=8.0$ and $1.3 \mathrm{~Hz}, 1 \mathrm{H}), 7.30$ (ddd apparent td, $J=7.8$ and $0.9 \mathrm{~Hz}, 1 \mathrm{H}$ ), 6.96 (ddd apparent td, $J=7.7$ and $1.0 \mathrm{~Hz}, 1 \mathrm{H}$ ), $2.23(\mathrm{~s}, 3 \mathrm{H}$ )

${ }^{13} \mathrm{C}$ NMR (100 MHz, $\left.\mathrm{CDCl}_{3}, 298 \mathrm{~K}\right) \delta 168.4,135.8,132.3,128.5,125.3,122.1,113.3,25.0$ 
<smiles>CC(=O)Nc1ccccc1F</smiles>

2'-Fluoroacetanilide (1c)

Prepared according to general procedure A, from 2-fluoroaniline (41\% yield).

Exhibited spectral data identical to a previous report. ${ }^{4}$

$\mathbf{R}_{\mathrm{f}} 0.4$ (petroleum ether/EtOAc 70:30)

${ }^{1} \mathrm{H}$ NMR (400 MHz, $\left.\mathrm{CDCl}_{3}, 298 \mathrm{~K}\right) \delta 8.29$ (ddd apparent td, $J=8.1$ and $\left.1.5 \mathrm{~Hz}, 1 \mathrm{H}\right), 7.42(\mathrm{br} \mathrm{s}, \mathrm{NH}), 7.14-7.01(\mathrm{~m}, 3 \mathrm{H})$, $2.22(\mathrm{~s}, 3 \mathrm{H})$

${ }^{19} \mathrm{~F}$ NMR $\left(376 \mathrm{MHz}, \mathrm{CDCl}_{3}, 298 \mathrm{~K}\right) \delta-131.4$

${ }^{13} \mathrm{C}$ NMR (100 MHz, $\left.\mathrm{CDCl}_{3}, 298 \mathrm{~K}\right) \delta 168.5,152.4\left(\mathrm{~d}, J_{C F}=243 \mathrm{~Hz}\right), 126.5\left(\mathrm{~d}, J_{C F}=10 \mathrm{~Hz}\right), 124.7\left(\mathrm{~d}, J_{C F}=4 \mathrm{~Hz}\right), 124.4$ $\left(\mathrm{d}, J_{C F}=8 \mathrm{~Hz}\right), 121.9,114.9\left(\mathrm{~d}, J_{C F}=19 \mathrm{~Hz}\right), 24.8$<smiles>CC(=O)Nc1ccccc1I</smiles>

\section{2'-lodoacetanilide (1d)}

Prepared according to general procedure A, from 2-iodoaniline (88\%).

Exhibited spectral data identical to a previous report. ${ }^{5}$

$\mathbf{R}_{\mathrm{f}} 0.3$ (petroleum ether/EtOAc 80:20)

${ }^{1} \mathrm{H}$ NMR $\left(400 \mathrm{MHz}, \mathrm{CDCl}_{3}, 298 \mathrm{~K}\right) \delta 8.21(\mathrm{~d}, J=7.9 \mathrm{~Hz}, 1 \mathrm{H}), 7.78(\mathrm{dd}, J=7.9$ and $1.0 \mathrm{~Hz}, 1 \mathrm{H}), 7.42(\mathrm{br} s, \mathrm{NH}), 7.35$ (ddd, apparent td, $J=7.9$ and $1.0 \mathrm{~Hz}, 1 \mathrm{H}$ ), 6.85 (dd apparent t, $J=7.3 \mathrm{~Hz}, 1 \mathrm{H}$ ), 2.25 (s, 3H)

${ }^{13} \mathrm{C}$ NMR (100 MHz, $\left.\mathrm{CDCl}_{3}, 298 \mathrm{~K}\right) \delta 168.4,138.9,138.3,129.4,126.1,122.2,90.1,25.0$<smiles>CC(=O)Nc1ccccc1Cl</smiles>

\section{2'-Chloroacetanilide (1e)}

Prepared according to general procedure A, from 2-chloroaniline (24\% yield).

Exhibited spectral data identical to a previous report. ${ }^{6}$

$\mathbf{R}_{\mathbf{f}} 0.4$ (petroleum ether/EtOAc 70:30)

${ }^{1} \mathrm{H}$ NMR (400 MHz, $\mathrm{CDCl}_{3}, 298 \mathrm{~K}$ ) $\delta 8.35$ (dd apparent br d, $J=8.1 \mathrm{~Hz}, 1 \mathrm{H}$ ), 7.65 (br s, NH), 7.36 (dd, $J=8.0$ and $1.4 \mathrm{~Hz}, 1 \mathrm{H}$ ), 7.26 (ddd apparent td, $J=7.9$ and $1.6 \mathrm{~Hz}, 1 \mathrm{H}$ ), 7.03 (ddd apparent td, $J=7.7$ and $1.0 \mathrm{~Hz}, 1 \mathrm{H}$ ), $2.24(\mathrm{~s}, 3 \mathrm{H}$ )

${ }^{13} \mathrm{C}$ NMR (100 MHz, $\left.\mathrm{CDCl}_{3}, 298 \mathrm{~K}\right) \delta$ 168.4, 134.7, 129.1, 127.8, 124.7, 122.7, 121.8, 25.0

(4) Naik, S.; Bhattacharjya, G.; Talukdar, B.; Patel, B. K. Eur. J. Org. Chem. 2004, 1254-1260.

(5) Kee, C. H.; Ariffin, A.; Awang, K.; Takeya, K.; Morita, H.; Hussain, S. I.; Chan, K. M.; Wood, P. J.; Threadgill, M. D.; Lim, C. G.; Ng, S. W.; Weber, J. F. F.; Thomas, N. F. Org. Biomol. Chem. 2010, 8, 5646-5660.

(6) Bedford, R. B.; Haddow, M. F.; Mitchell, C. J.; Webster, R. L. Angew. Chem. Int. Ed. 2011, 50, 5524-5527. 
<smiles>CC(=O)Nc1ccccc1C(F)(F)F</smiles>

\section{2'-Trifluoromethylacetanilide (1f)}

Prepared according to a literature procedure, ${ }^{7}$ from 2 -trifluoromethylaniline (86\% yield).

Exhibited spectral data identical to a previous report. ${ }^{8}$

$\mathbf{R}_{\mathbf{f}} 0.3$ (petroleum ether/EtOAc 70:30)

${ }^{1} \mathrm{H}$ NMR $\left(400 \mathrm{MHz}, \mathrm{CDCl}_{3}, 298 \mathrm{~K}\right) \delta 8.14(\mathrm{~d}, J=8.1 \mathrm{~Hz}, 1 \mathrm{H}), 7.60(\mathrm{~d}, J=7.9 \mathrm{~Hz}, 1 \mathrm{H}), 7.55$ (dd apparent t, $J=7.9 \mathrm{~Hz}$, $1 \mathrm{H}$ ), 7.44 (br s, NH), 7.23 (dd apparent $t, J=7.6 \mathrm{~Hz}, 1 \mathrm{H}$ ), 2.21 (s, 3H)

${ }^{19} \mathrm{~F}$ NMR (376 MHz, $\left.\mathrm{CDCl}_{3}, 298 \mathrm{~K}\right) \delta-60.6$

${ }^{13} \mathrm{C}$ NMR (100 MHz, $\mathrm{CDCl}_{3}, 298 \mathrm{~K}$ ) $\delta 168.6,135.3$ (q apparent d, $J_{C F}=1 \mathrm{~Hz}$ ), 133.0, 126.1 (q, $J_{C F}=5 \mathrm{~Hz}$ ), 124.9, 124.7, 124.2 (q apparent d, $J_{C F}=273 \mathrm{~Hz}$ ), $120.4\left(\mathrm{~d}, J_{C F}=30 \mathrm{~Hz}\right.$ ), 24.8<smiles>Cc1ccccc1NC(=O)c1ccccc1</smiles>

\section{2'-Acetylacetanilide (19)}

Prepared according to general procedure A, from 2-acetylaniline (66\%).

Exhibited spectral data identical to a previous report. ${ }^{9}$

$\mathbf{R}_{\mathrm{f}} 0.5$ (petroleum ether/EtOAc 70:30)

${ }^{1}$ H NMR (400 MHz, $\left.\mathrm{CDCl}_{3}, 298 \mathrm{~K}\right) \delta 11.70(\mathrm{br} \mathrm{s}, \mathrm{NH}), 8.74(\mathrm{~d}, J=8.5 \mathrm{~Hz}, 1 \mathrm{H}), 7.90(\mathrm{~d}, J=8.0 \mathrm{~Hz}, 1 \mathrm{H}), 7.55(\mathrm{dd}$ apparent t, $J=7.9 \mathrm{~Hz}, 1 \mathrm{H}), 7.12(\mathrm{dd}$, apparent t, $J=7.6 \mathrm{~Hz}, 1 \mathrm{H}), 2.67(\mathrm{~s}, 3 \mathrm{H}), 2.23(\mathrm{~s}, 3 \mathrm{H})$

${ }^{13}$ C NMR (100 MHz, CDCl $_{3}, 298$ K) $\delta 202.9,169.5,141.0,135.2,131.6,122.3,121.7,120.7,28.7,25.6$<smiles>CC(=O)Nc1ccccc1C</smiles>

\section{2'-Methylacetanilide (1h)}

Prepared according to general procedure B, from 2-methylaniline (26\% yield).

Exhibited spectral data identical to a previous report. ${ }^{10}$

$\mathbf{R}_{\mathrm{f}} 0.2$ (petroleum ether/EtOAc 70:30)

${ }^{1} \mathrm{H}$ NMR (400 MHz, $\left.\mathrm{CDCl}_{3}, 298 \mathrm{~K}\right) \delta 7.72(\mathrm{~d}, J=8.0 \mathrm{~Hz}, 1 \mathrm{H}), 7.21-7.06(\mathrm{~m}, 4 \mathrm{H}), 2.25(\mathrm{~s}, 3 \mathrm{H}), 2.18(\mathrm{~s}, 3 \mathrm{H})$

${ }^{13} \mathrm{C}$ NMR (100 MHz, $\left.\mathrm{CDCl}_{3}, 298 \mathrm{~K}\right) \delta 168.5,135.8,130.6,129.6,126.8,125.5,123.7,24.4,17.9$ 
<smiles>CC(=O)Nc1cccc(I)c1</smiles>

\section{3'-lodoacetanilide (1i)}

Prepared according to general procedure B, from 3-iodoaniline (55\% yield).

Exhibited spectral data identical to a previous report. ${ }^{11}$

$\mathbf{R}_{\mathbf{f}} 0.2$ (petroleum ether/EtOAc 70:30)

${ }^{1} \mathrm{H}$ NMR (400 MHz, $\mathrm{CDCl}_{3}, 298 \mathrm{~K}$ ) $\delta 7.91$ (dd apparent $\mathrm{t}, J=1.8 \mathrm{~Hz}, 1 \mathrm{H}$ ), 7.88 (br s, NH), 7.46 (ddd, $J=8.2,2.0$ and $0.8 \mathrm{~Hz}, 1 \mathrm{H}$ ), 7.41 (ddd apparent dt, $J=7.9$ and $1.2 \mathrm{~Hz}, 1 \mathrm{H}$ ), 7.01 (dd apparent t, $J=8.0 \mathrm{~Hz}, 1 \mathrm{H}$ ), $2.16(\mathrm{~s}, 3 \mathrm{H})$

${ }^{13} \mathrm{C}$ NMR (100 MHz, $\left.\mathrm{CDCl}_{3}, 298 \mathrm{~K}\right) \delta 168.9,139.2,133.4,130.5,128.8,119.3,94.2,24.7$<smiles>CC(=O)Nc1cccc(Br)c1</smiles>

\section{3'-Bromoacetanilide (1j)}

Prepared according to general procedure B, from 3-bromoaniline (59\% yield)

Exhibited spectral data identical to a previous report. ${ }^{12}$

$\mathbf{R}_{\mathrm{f}} 0.25$ (petroleum ether/EtOAc 70:30)

${ }^{1} \mathrm{H}$ NMR (400 MHz, $\left.\mathbf{C D C l}_{3}, 298 \mathrm{~K}\right) \delta 7.81(\mathrm{br} \mathrm{s}, \mathrm{NH}), 7.76$ (dd apparent t, $\left.J=1.9 \mathrm{~Hz}, 1 \mathrm{H}\right), 7.40(\mathrm{dd}, J=8.0 \mathrm{and} 0.7 \mathrm{~Hz}$, $1 \mathrm{H}$ ), 7.22 (ddd apparent dt, $J=8.2$ and $1.4 \mathrm{~Hz}, 1 \mathrm{H}$ ), 7.15 (dd apparent t, $J=8.0 \mathrm{~Hz}, 1 \mathrm{H}$ ), $2.17(\mathrm{~s}, 3 \mathrm{H}$ )

${ }^{13} \mathrm{C}$ NMR (100 MHz, $\left.\mathrm{CDCl}_{3}, 298 \mathrm{~K}\right) \delta 168.9,139.3,130.4,127.38,123.0,122.7,118.5,24.7$<smiles>CC(=O)Nc1cccc(Cl)c1</smiles>

\section{3'-Chloroacetanilide (1k)}

Prepared according to general procedure B, from 3-chloroaniline (61\% yield).

Exhibited spectral data identical to a previous report. ${ }^{13}$

$\mathbf{R}_{\mathrm{f}} 0.2$ (petroleum ether/EtOAc 70:30)

${ }^{1} \mathrm{H}$ NMR (400 MHz, $\mathrm{CDCl}_{3}, 298 \mathrm{~K}$ ) $\delta 8.09$ (br s, NH), 7.64 (dd apparent t, $J=2.0 \mathrm{~Hz}, 1 \mathrm{H}$ ), 7.33 (ddd, $J=8.1,2.0$ and $0.9 \mathrm{~Hz}, 1 \mathrm{H}$ ), 7.20 (dd apparent t, $J=8.1 \mathrm{~Hz}, 1 \mathrm{H}$ ), 7.06 (ddd, $J=8.0,2.0$ and $1.0 \mathrm{~Hz}, 1 \mathrm{H}), 2.17(\mathrm{~s}, 3 \mathrm{H})$

${ }^{13} \mathrm{C}$ NMR (100 MHz, $\left.\mathrm{CDCl}_{3}, 298 \mathrm{~K}\right) \delta 169.0,139.2,134.6,130.0,124.4,120.2,118.0,24.6$ 
<smiles>CC(=O)Nc1cccc(F)c1</smiles>

\section{3'-Fluoroacetanilide (1I)}

Prepared according to general procedure B, from 3-fluoroaniline (71\% yield).

Exhibited spectral data identical to a previous report. ${ }^{11}$

$\mathbf{R}_{\mathbf{f}} 0.25$ (petroleum ether/EtOAc 70:30)

${ }^{1} \mathrm{H}$ NMR (400 MHz, $\mathrm{CDCl}_{3}, 298 \mathrm{~K}$ ) $\delta 8.00$ (br s, NH), 7.48 (ddd apparent dt, $J=11.0$ and $2.2 \mathrm{~Hz}, 1 \mathrm{H}$ ), 7.23 (ddd apparent td, $J=8.1$ and $6.4 \mathrm{~Hz}, 1 \mathrm{H}$ ), 7.14 (ddd, $J=8.1,1.8$ and $0.9 \mathrm{~Hz}, 1 \mathrm{H}$ ), 6.79 (dddd apparent tdd, $J=8.3,2.5$ and $0.9 \mathrm{~Hz}$, $1 \mathrm{H}), 2.17(\mathrm{~s}, 3 \mathrm{H})$

${ }^{19} \mathrm{~F}$ NMR (376 MHz, $\left.\mathrm{CDCl}_{3}, 298 \mathrm{~K}\right) \delta-111.5$

${ }^{13} \mathrm{C}$ NMR (100 MHz, $\left.\mathrm{CDCl}_{3}, 298 \mathrm{~K}\right) \delta 169.0,163.1\left(\mathrm{~d}, J_{C F}=245 \mathrm{~Hz}\right), 139.6\left(\mathrm{~d}, J_{C F}=11 \mathrm{~Hz}\right), 130.1\left(\mathrm{~d}, J_{C F}=9 \mathrm{~Hz}\right), 115.3$ $\left(\mathrm{d}, J_{C F}=3 \mathrm{~Hz}\right), 111.1\left(\mathrm{~d}, J_{C F}=21 \mathrm{~Hz}\right), 107.5\left(\mathrm{~d}, J_{C F}=26 \mathrm{~Hz}\right), 24.6$<smiles>CC(=O)Nc1cccc(C(C)=O)c1</smiles>

\section{3'-Acetylacetanilide (1m)}

Prepared according to general procedure B, from 3-acetylaniline (72\% yield).

Exhibited spectral data identical to a previous report. ${ }^{14}$

$\mathbf{R}_{\mathrm{f}} 0.1$ (petroleum ether/ EtOAc 70:30)

${ }^{1} \mathrm{H}$ NMR (400 MHz, $\mathbf{C D C l}_{3}, 298 \mathrm{~K}$ ) $\delta 8.17(\mathrm{br} \mathrm{s}, \mathrm{NH}), 8.03$ (dd apparent t, $J=1.9 \mathrm{~Hz}, 1 \mathrm{H}$ ), 7.97 (ddd, $J=8.1,2.2$ and $0.9 \mathrm{~Hz}, 1 \mathrm{H}$ ), 7.67 (ddd, $J=7.8,1.5$ and $1.1 \mathrm{~Hz}, 1 \mathrm{H}), 7.41$ (dd apparent t, $J=7.9 \mathrm{~Hz}, 1 \mathrm{H}), 2.59(\mathrm{~s}, 3 \mathrm{H}), 2.21(\mathrm{~s}, 3 \mathrm{H})$

${ }^{13}$ C NMR (100 MHz, $\left.\mathrm{CDCl}_{3}, 298 \mathrm{~K}\right) \delta 198.4,169.1,138.8,137.7,129.5,124.8,124.3,119.3,26.9,24.7$<smiles>COc1cccc(NC(C)=O)c1</smiles>

\section{Methyl 3-acetamidobenzoate (1n)}

Prepared according to general procedure B, from methyl 3-aminobenzoate (76\%).

Exhibited spectral data identical to a previous report. ${ }^{15}$

$\mathbf{R}_{\mathbf{f}} 0.15$ (petroleum ether/EtOAc 70:30)

${ }^{1} \mathrm{H}$ NMR (400 MHz, $\mathbf{C D C l}_{3}, 298 \mathrm{~K}$ ) $\delta 8.05$ (br s, NH), 8.05 (dd apparent t, $J=1.8 \mathrm{~Hz}, 1 \mathrm{H}$ ), 7.89 (ddd, $J=8.1,2.1$ and $0.9 \mathrm{~Hz}, 1 \mathrm{H}$ ), 7.75 (ddd apparent dt, $J=7.8$ and $1.2 \mathrm{~Hz}, 1 \mathrm{H}$ ), 7.36 (dd apparent t, $J=7.9 \mathrm{~Hz}, 1 \mathrm{H}$ ), $3.88(\mathrm{~s}, 3 \mathrm{H}), 2.19(\mathrm{~s}$, $3 \mathrm{H})$

${ }^{13} \mathrm{C}$ NMR (100 MHz, $\left.\mathrm{CDCl}_{3}, 298 \mathrm{~K}\right) \delta 169.0,166.9,138.4,130.8,129.2,125.3,124.6,120.9,52.4,24.6$ 
<smiles>CC(=O)Nc1cccc(S(N)(=O)=O)c1</smiles>

\section{3'-(Trimethylsilyl)acetanilide (10)}

Prepared according to literature procedures, ${ }^{16,17}$ from 3 -chloroaniline (30\%, 2 steps).

Exhibited spectral data identical to a previous report. ${ }^{17}$

$\mathbf{R}_{\mathbf{f}} 0.2$ (petroleum ether/EtOAc 80:20)

${ }^{1} \mathrm{H}$ NMR (400 MHz, $\mathbf{C D C l}_{3}, 298 \mathrm{~K}$ ) $\delta 7.63$ (ddd, $J=7.9,2.2$ and $1.3 \mathrm{~Hz}, 1 \mathrm{H}$ ), 7.49 (dd apparent br d, $J=1.9 \mathrm{~Hz}, 1 \mathrm{H}$ ), 7.40 (br s, NH), 7.31 (dd apparent t, $J=7.6 \mathrm{~Hz}, 1 \mathrm{H}$ ), 7.25 (d, $J=7.2 \mathrm{~Hz}, 1 \mathrm{H}$ ), 2.17 (s, 3H), 0.25 (s, 9H)

${ }^{13} \mathrm{C}$ NMR (100 MHz, $\left.\mathrm{CDCl}_{3}, 298 \mathrm{~K}\right) \delta$ 168.5, 141.7, 137.4, 129.4, 128.6, 124.6, 120.8, 24.7, -1.1<smiles>CC(=O)Nc1cccc(C)c1</smiles>

\section{3'-Methylacetanilide (1p)}

Prepared according to general procedure A, from 3-methylaniline (95\%).

Exhibited spectral data identical to a previous report. ${ }^{18}$

$\mathbf{R}_{\mathfrak{f}} 0.2$ (petroleum ether/EtOAc 70:30)

${ }^{1} \mathrm{H}$ NMR $\left(400 \mathrm{MHz}, \mathrm{CDCl}_{3}, 298 \mathrm{~K}\right) \delta 7.61(\mathrm{br} \mathrm{s}, \mathrm{NH}), 7.36(\mathrm{~s}, 1 \mathrm{H}), 7.28(\mathrm{~d}, J=8.5 \mathrm{~Hz}, 1 \mathrm{H}), 7.18$ (dd apparent t, $J=7.8 \mathrm{~Hz}, 1 \mathrm{H}), 6.91$ (dd, $J=7.5$ and $0.3 \mathrm{~Hz}, 1 \mathrm{H}), 2.31(\mathrm{~s}, 3 \mathrm{H}), 2.15(\mathrm{~s}, 3 \mathrm{H})$

${ }^{13} \mathrm{C}$ NMR (100 MHz, $\left.\mathrm{CDCl}_{3}, 298 \mathrm{~K}\right) \delta 168.7,138.9,137.9,128.9,125.2,120.7,117.1,24.7,21.6$<smiles>COc1cccc(NC(C)=O)c1</smiles>

\section{3'-Methoxyacetanilide (1q)}

Prepared according to general procedure A, from 3-methoxyaniline (76\%).

Exhibited spectral data identical to a previous report. ${ }^{19}$

$\mathbf{R}_{\mathfrak{f}} 0.25$ (petroleum ether/EtOAc 60:40)

${ }^{1} \mathrm{H}$ NMR (400 MHz, $\mathbf{C D C l}_{3}, 298 \mathrm{~K}$ ) $\delta 7.85$ (br s, NH), 7.27 (dd apparent t, J=2.2 Hz, 1H), 7.18 (dd apparent $\mathrm{t}$, $J=8.1 \mathrm{~Hz}, 1 \mathrm{H}), 6.99(\mathrm{dd}, J=8.0$ and $1.1 \mathrm{~Hz}, 1 \mathrm{H}), 6.64(\mathrm{ddd}, J=8.3,2.5$ and $0.7 \mathrm{~Hz}, 1 \mathrm{H}), 3.76(\mathrm{~s}, 3 \mathrm{H}), 2.14(\mathrm{~s}, 3 \mathrm{H})$

${ }^{13}$ C NMR (100 MHz, $\left.\mathrm{CDCl}_{3}, 298 \mathrm{~K}\right) \delta 168.9,160.1,139.3,129.7,112.2,110.0,105.8,55.3,24.7$

(18) Taylor, J. E.; Jones, M. D.; Wiliams, J. M. J.; Bull, S. D. J. Org. Chem. 2012, 77, 2808-2018

(19) Beharry, A. A.; Sadovski, O.; Woolley, G. A. J. Am. Chem. Soc. 2011, 133, 19684-19687. 
<smiles>CC(=O)Nc1cccc(OC(C)=O)c1</smiles>

\section{3-Acetamidophenyl acetate (1r)}

Prepared according to a literature procedure,$^{20}$ from 3-aminophenol (98\%).

Exhibited spectral data identical to a previous report. ${ }^{21}$

$\mathbf{R}_{\mathrm{f}} 0.3\left(\mathrm{CH}_{2} \mathrm{Cl}_{2} / \mathrm{MeOH} 97: 3\right)$

${ }^{1} \mathbf{H}$ NMR (400 MHz, $\left.\mathbf{C D C l}_{3}, 298 \mathrm{~K}\right) \delta 7.77(\mathrm{br} \mathrm{s}, \mathrm{NH}), 7.47$ (dd, apparent t, J=2.1 Hz, 1H), 7.25 (dd apparent t, $J=8.1 \mathrm{~Hz}, 1 \mathrm{H}$ ), 7.13 (ddd, $J=8.1,2.0$ and $0.9 \mathrm{~Hz}, 1 \mathrm{H}$ ), 6.79 (ddd, $J=8.1,2.2$ and $0.9 \mathrm{~Hz}, 1 \mathrm{H}$ ), $2.30(\mathrm{~s}, 3 \mathrm{H}), 2.07(\mathrm{~s}$, $3 \mathrm{H})$

${ }^{13} \mathrm{C}$ NMR (100 MHz, $\left.\mathrm{CDCl}_{3}, 298 \mathrm{~K}\right) \delta 170.1,168.8,151.0,139.4,129.6,117.1,113.4,24.6,21.3$<smiles>CC(C)(C)C(=O)Nc1ccccc1</smiles>

\section{N-Pivaloylaniline (4a)}

Prepared according to general procedure A (using pivaloyl chloride instead of acetic anhydride), from aniline (83\%).

Exhibited spectral data identical to a previous report. ${ }^{22}$

$\mathbf{R}_{\mathbf{f}} 0.4$ (petroleum ether/EtOAc $90: 10$ )

${ }^{1} \mathrm{H}$ NMR (400 MHz, $\left.\mathrm{CDCl}_{3}, 298 \mathrm{~K}\right) \delta 7.53(\mathrm{dd}, J=8.6$ and $1.1 \mathrm{~Hz}, 2 \mathrm{H}), 7.32(\mathrm{br} \mathrm{s}, \mathrm{NH}), 7.32(\mathrm{dd}, J=8.5 \mathrm{and} 7.5 \mathrm{~Hz}, 2 \mathrm{H})$, $7.10(\mathrm{tt}, J=7.4$ and $1.0 \mathrm{~Hz}, 1 \mathrm{H}), 1.32(\mathrm{~s}, 9 \mathrm{H})$

${ }^{13} \mathrm{C}$ NMR (100 MHz, $\left.\mathrm{CDCl}_{3}, 298 \mathrm{~K}\right) \delta 176.7,138.1,129.1,124.3,120.0,39.7,27.8$<smiles>O=C(Nc1ccccc1)OCc1ccccc1</smiles>

\section{Benzyl phenylcarbamate (4c)}

Prepared according to a literature procedure ${ }^{23}$ from aniline (82\%).

Exhibited spectral data identical to a previous report. ${ }^{24}$

$\mathbf{R}_{\mathrm{f}} 0.6$ (petroleum ether/EtOAc $90: 10$ )

${ }^{1} \mathrm{H}$ NMR $\left(400 \mathrm{MHz} ; \mathrm{CDCl}_{3}\right) \delta$ 7.42-7.33 (m, 7H), 7.32-7.27 (m, 2H), 7.06 (ddt, J= 7.5, 7.2 and 1.2 Hz, $\left.1 \mathrm{H}\right), 6.67$ (br s, $\mathrm{NH}), 5.20(\mathrm{~s}, 2 \mathrm{H})$

${ }^{13} \mathrm{C}$ NMR $\left(100 \mathrm{MHz}, \mathrm{CDCl}_{3}\right) \delta 153.4,137.9,136.1,129.2,128.7,128.48,128.44,123.6,118.8,67.1$

(20) Guckian, K. M.; Caldwell, R. D.; Kumaravel, G.; Lee, W.-C.; Lin, E. Y.-S.; Liu, X.; Ma, B.; Scott, D. M.; Shi, Z.; Zheng, G. Z.; Taveras, A. G.; Thomas, J. WO 2010/051031 Patent, 2010.

(21) Ishikawa, T.; Arai, M.; Nishiuchi, H.; Ishiguro, H.; Saito, S.; Chem. Lett. 2008, 37, 1066-1067.

(22) Ma, F.; Xie, X.; Zhang, L.; Peng, Z.; Ding, L.; Fu, L.; Zhang, Z. J. Org. Chem. 2012, 77, 5279-5285.

(23) Wipf, P.; Maciejewski, J. P. Org. Lett. 2008, 10, 4383-4386.

(24) Lebel, H.; Leogane, O.; Org Lett. 2006, 8, 5717-5720. 


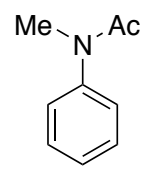

\section{N-Methylacetanilide (4f)}

Prepared according to a literature procedure, ${ }^{25}$ from acetanilide (25\%).

Exhibited spectral data identical to a previous report. ${ }^{25}$

$\mathbf{R}_{\mathfrak{f}} 0.3$ (petroleum ether/EtOAc 60:40)

${ }^{1} \mathrm{H}$ NMR (400 MHz, CDCl $\left.3,298 \mathrm{~K}\right) \delta 7.42(\mathrm{t}, J=7.5 \mathrm{~Hz}, 2 \mathrm{H}), 7.34(\mathrm{t}, J=7.4 \mathrm{~Hz}, 1 \mathrm{H}), 7.19(\mathrm{~d}, J=7.1 \mathrm{~Hz}, 2 \mathrm{H}), 3.27(\mathrm{~s}$, $3 \mathrm{H}), 1.87(\mathrm{~s}, 3 \mathrm{H})$

${ }^{13} \mathrm{C}$ NMR (100 MHz, $\left.\mathrm{CDCl}_{3}, 298 \mathrm{~K}\right) \delta 170.7,144.7,129.8,127.8,127.2,37.3,22.6$ 


\section{Synthesis and Characterization of Aryltriflates 2a-r}

\section{General Procedure C}

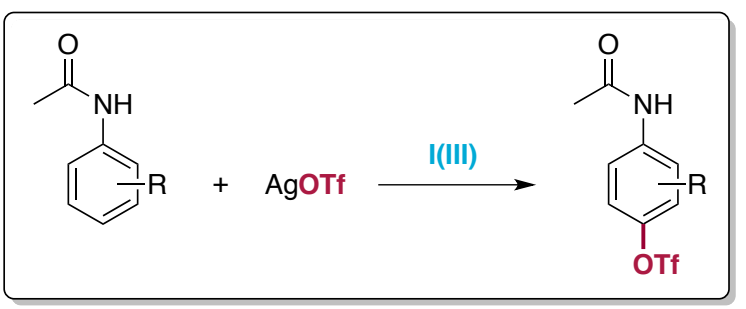

To a solution of acetanilide 1 ( $0.5 \mathrm{mmol}, 1.0$ equiv) and AgOTf (or AgONf) (0.6 mmol, 1.2 equiv) in DCE (4 mL, $0.125 \mathrm{M}$ ) in a $4 \mathrm{~mL}$ screw-cap vial under argon is added [bis(trifluoroacetoxy)iodo]benzene (BTI) $(0.75 \mathrm{mmol}, 1.5$ equiv). The resulting mixture is stirred for 1 hour at $20^{\circ} \mathrm{C}$ or $50^{\circ} \mathrm{C}$ then filtered over a short plug of Celite ${ }^{\circledR}$ and concentrated under reduced pressure. The crude product is purified by silica gel flash chromatography.<smiles>CC(=O)Nc1ccc(Oc2ccccc2)cc1</smiles>

\section{4-acetamidophenyl trifluoromethanesulfonate (2a)}

Prepared according to general procedure $\mathrm{C}\left(20^{\circ} \mathrm{C}\right)$, from acetanilide $1 \mathrm{a}(66 \%$, white powder).

Exhibited spectral data identical to a previous report. ${ }^{26}$

\section{Large-scale procedure}

To a solution of acetanilide 1a (676 mg, $5.00 \mathrm{mmol}, 1.00$ equiv) and AgOTf $(1.54 \mathrm{~g}, 6.00 \mathrm{mmol}, 1.20$ equiv) in DCE $(40 \mathrm{~mL}, 0.125 \mathrm{M})$ in a Schlenk flask under argon is added [bis(trifluoroacetoxy)iodo]benzene (BTI) $(3.22 \mathrm{~g}, 7.20 \mathrm{mmol}$, 1.50 equiv). The resulting mixture is stirred for 1 hour at $20^{\circ} \mathrm{C}$. Water is then added and the crude product is extracted with $\mathrm{CH}_{2} \mathrm{Cl}_{2}$, washed with a saturated solution of $\mathrm{NaCl}$, dried with $\mathrm{MgSO}_{4}$ and concentrated under reduced pressure. The crude product is purified by silica gel flash chromatography to afford $793 \mathrm{mg}$ of aryltriflate $\mathbf{2 a}(56 \%)$ as a white powder.

mp $138-140{ }^{\circ} \mathrm{C}$

$\mathbf{R}_{\mathbf{f}} 0.25$ (petroleum ether/EtOAc 75:25)

${ }^{1} \mathrm{H}$ NMR (400 MHz, $\left.\mathrm{CDCl}_{3}, 298 \mathrm{~K}\right) \delta 7.60(\mathrm{~d}, J=9.1 \mathrm{~Hz}, 2 \mathrm{H}), 7.35(\mathrm{br} \mathrm{s}, \mathrm{NH}), 7.23(\mathrm{~d}, J=9.1 \mathrm{~Hz}, 2 \mathrm{H}), 2.20(\mathrm{~s}, 3 \mathrm{H})$

${ }^{19} \mathrm{~F}$ NMR (376 $\left.\mathrm{MHz}, \mathrm{CDCl}_{3}, 298 \mathrm{~K}\right) \delta-72.7$

${ }^{13} \mathrm{C}$ NMR (100 MHz, $\left.\mathrm{CDCl}_{3}, 298 \mathrm{~K}\right) \delta 168.6,145.4,138.1,122.1,121.2,118.9$ (q, J JF $\left.=321 \mathrm{~Hz}\right), 24.7$

HRMS Calculated for $\mathrm{C}_{9} \mathrm{H}_{8} \mathrm{~F}_{3} \mathrm{NO}_{4} \mathrm{~S}[\mathrm{M}+\mathrm{H}]^{+} 284.0199$, found 284.0190 
<smiles>CC(=O)Nc1ccc(Br)cc1Br</smiles>

4-acetamido-3-bromophenyl trifluoromethanesulfonate (2b)

Prepared according to general procedure $C\left(20^{\circ} \mathrm{C}\right)$, from acetanilide $1 \mathrm{~b}(71 \%$, white powder).

Exhibited spectral data identical to a previous report. ${ }^{27}$

mp $91-92^{\circ} \mathrm{C}$

$\mathbf{R}_{\mathbf{f}} 0.15$ (petroleum ether/EtOAc 90:10)

${ }^{1} \mathrm{H}$ NMR $\left(400 \mathrm{MHz}, \mathrm{CDCl}_{3}, 298 \mathrm{~K}\right) \delta 8.51(\mathrm{~d}, J=9.2 \mathrm{~Hz}, 1 \mathrm{H}), 7.63(\mathrm{br} \mathrm{s}, \mathrm{NH}), 7.50(\mathrm{~d}, J=2.8 \mathrm{~Hz}, 1 \mathrm{H}), 7.26(\mathrm{dd}, J=9.2$ and $2.8 \mathrm{~Hz}, 1 \mathrm{H}), 2.26(\mathrm{~s}, 3 \mathrm{H})$

${ }^{19} \mathrm{~F}$ NMR (376 $\left.\mathrm{MHz}, \mathrm{CDCl}_{3}, 298 \mathrm{~K}\right) \delta-72.6$

${ }^{13} \mathrm{C}$ NMR (100 MHz, $\left.\mathrm{CDCl}_{3}, 298 \mathrm{~K}\right) \delta 168.5,144.5,136.2,125.3,122.4,121.5,118.7$ (q, $\left.J_{C F}=321 \mathrm{~Hz}\right), 113.0,25.0$

HRMS Calculated for $\mathrm{C}_{9} \mathrm{H}_{7} \mathrm{BrF}_{3} \mathrm{NO}_{4} \mathrm{~S}[\mathrm{M}+\mathrm{H}]^{+} 361.9304$, found 361.9295<smiles>CC(=O)Nc1ccc(O)cc1F</smiles>

4-acetamido-3-fluorophenyl trifluoromethanesulfonate (2c)

Prepared according to general procedure $\mathrm{C}\left(20^{\circ} \mathrm{C}\right)$, from acetanilide $1 \mathrm{c}(60 \%$, brown powder).

mp $127-129{ }^{\circ} \mathrm{C}$

$\mathbf{R}_{\mathfrak{f}} 0.3$ (petroleum ether/EtOAc 70:30)

${ }^{1} \mathrm{H}$ NMR (400 MHz, $\mathrm{CDCl}_{3}, 298 \mathrm{~K}$ ) $\delta 8.48$ (dd apparent t, $\left.J=9.1 \mathrm{~Hz}, 1 \mathrm{H}\right), 7.39$ (br s, NH), 7.11-7.07 (m, 2H), $2.25(\mathrm{~s}, 3 \mathrm{H})$ ${ }^{19} \mathrm{~F}$ NMR (376 MHz, $\left.\mathrm{CDCl}_{3}, 298 \mathrm{~K}\right) \delta-72.6(3 \mathrm{~F}),-126.4(1 \mathrm{~F})$

${ }^{13} \mathrm{C}$ NMR (100 MHz, $\left.\mathbf{C D C l}_{3}, 298 \mathrm{~K}\right) \delta 168.5\left(\mathrm{~d}, J_{C F}=4 \mathrm{~Hz}\right), 151.7\left(\mathrm{~d}, J_{C F}=248 \mathrm{~Hz}\right), 144.2\left(\mathrm{~d}, J_{C F}=11 \mathrm{~Hz}\right), 127.0(\mathrm{~d}$, $\left.J_{C F}=10 \mathrm{~Hz}\right), 122.4\left(\mathrm{~d}, J_{C F}=2 \mathrm{~Hz}\right), 118.9\left(\mathrm{q}, J_{C F}=321 \mathrm{~Hz}\right), 117.8\left(\mathrm{~d}, J_{C F}=4 \mathrm{~Hz}\right), 109.5\left(\mathrm{~d}, J_{C F}=24 \mathrm{~Hz}\right), 24.7$

HRMS Calculated for $\mathrm{C}_{9} \mathrm{H}_{7} \mathrm{~F}_{4} \mathrm{NO}_{4} \mathrm{~S}[\mathrm{M}+\mathrm{H}]^{+} 302.0105$, found 302.0091<smiles>CC(=O)Nc1ccc(O)cc1I</smiles>

4-acetamido-3-iodophenyl trifluoromethanesulfonate (2d)

Prepared according to general procedure $\mathrm{C}\left(20^{\circ} \mathrm{C}\right)$, from acetanilide $1 \mathrm{~d}(46 \%$, yellow powder).

mp $123-125^{\circ} \mathrm{C}$

$\mathbf{R}_{\mathrm{f}} 0.3$ (petroleum ether/EtOAc 70:30)

${ }^{1} \mathrm{H}$ NMR $\left(400 \mathrm{MHz}, \mathrm{CDCl}_{3}, 298 \mathrm{~K}\right) \delta 8.36(\mathrm{~d}, J=9.2 \mathrm{~Hz}, 1 \mathrm{H}), 7.69(\mathrm{~d}, J=2.8 \mathrm{~Hz}, 1 \mathrm{H}), 7.48(\mathrm{br} \mathrm{s}, \mathrm{NH}), 7.29(\mathrm{dd}, J=9.2$ and $2.8 \mathrm{~Hz}, 1 \mathrm{H}), 2.26(\mathrm{~s}, 3 \mathrm{H})$

${ }^{19} \mathrm{~F}$ NMR (376 $\left.\mathrm{MHz}, \mathrm{CDCl}_{3}, 298 \mathrm{~K}\right) \delta-72.6$

${ }^{13} \mathrm{C}$ NMR (100 MHz, $\left.\mathrm{CDCl}_{3}, 298 \mathrm{~K}\right) \delta 168.5,144.8,138.7,131.5,122.3,122.1,118.8$ (q, $\left.J_{C F}=321 \mathrm{~Hz}\right), 88.8,25.0$

HRMS Calculated for $\mathrm{C}_{9} \mathrm{H}_{7} \mathrm{~F}_{3} \mathrm{INO}_{4} \mathrm{~S}[\mathrm{M}+\mathrm{H}]^{+} 409.9165$, found 409.9157 
<smiles>CC(=O)Nc1ccc(O)cc1Cl</smiles>

\section{4-Acetamido-3-chlorophenyl trifluoromethanesulfonate (2e)}

Prepared according to general procedure $\mathrm{C}\left(20^{\circ} \mathrm{C}\right)$, from acetanilide $1 \mathrm{e}(84 \%$, light grey powder).

mp $77-79^{\circ} \mathrm{C}$

$\mathbf{R}_{\mathfrak{f}} 0.3$ (petroleum ether/EtOAc 70:30)

${ }^{1}$ H NMR (400 MHz, CDCl $\left.3,298 \mathrm{~K}\right) \delta 8.54(\mathrm{~d}, J=9.3 \mathrm{~Hz}, 1 \mathrm{H}), 7.63(\mathrm{br} \mathrm{s}, \mathrm{NH}), 7.35(\mathrm{~d}, J=2.8 \mathrm{~Hz}, 1 \mathrm{H}), 7.21(\mathrm{dd}, J=9.2$ and $2.8 \mathrm{~Hz}, 1 \mathrm{H}), 2.27(\mathrm{~s}, 3 \mathrm{H})$

${ }^{19} \mathrm{~F}$ NMR (376 MHz, $\left.\mathrm{CDCl}_{3}, 298 \mathrm{~K}\right) \delta-72.6$

${ }^{13} \mathrm{C}$ NMR (100 MHz, $\left.\mathrm{CDCl}_{3}, 298 \mathrm{~K}\right) \delta$ 168.5, 144.4, 135.1, 123.0, 122.36, 122.23, 121.0, 118.8 (q apparent d, $\left.J_{C F}=321 \mathrm{~Hz}\right), 25.1$

HRMS Calculated for $\mathrm{C}_{9} \mathrm{H}_{7} \mathrm{ClF}_{3} \mathrm{NO}_{4} \mathrm{~S}[\mathrm{M}+\mathrm{H}]^{+} 317.9809$, found 317.9815<smiles>CC(=O)Nc1ccc(O)cc1C(F)(F)F</smiles>

4-Acetamido-3-(trifluoromethyl)phenyl trifluoromethanesulfonate (2f)

Prepared according to general procedure $\mathrm{C}\left(50^{\circ} \mathrm{C}\right)$, from acetanilide $1 \mathrm{f}(45 \%$, white powder $)$.

mp $98-100^{\circ} \mathrm{C}$

$\mathbf{R}_{\mathrm{f}} 0.3$ (petroleum ether/EtOAc 70:30)

${ }^{1} \mathrm{H}$ NMR $\left(400 \mathrm{MHz}, \mathrm{CDCl}_{3}, 298 \mathrm{~K}\right) \delta 8.42(\mathrm{~d}, J=9.1 \mathrm{~Hz}, 1 \mathrm{H}), 7.51(\mathrm{~d}, J=2.9 \mathrm{~Hz}, 1 \mathrm{H}), 7.47(\mathrm{dd}, J=9.1$ and $2.9 \mathrm{~Hz}, 1 \mathrm{H})$, 7.45 (br s, NH), 2.25 (s, 3H)

${ }^{19} \mathrm{~F}$ NMR (376 MHz, $\left.\mathrm{CDCl}_{3}, 298 \mathrm{~K}\right) \delta-61.2\left(\mathrm{ArCF}_{3}\right),-72.5\left(\mathrm{ArOSO}_{2} \mathrm{CF}_{3}\right)$

${ }^{13} \mathrm{C}$ NMR (63 MHz, $\left.\mathbf{C D C l}_{3}, 298 \mathrm{~K}\right) \delta 168.5,144.9,135.6$ (q apparent $\mathrm{d}, J_{C F}=1 \mathrm{~Hz}$ ), 126.2, 126.0, 122.9 (q, $J_{C F}=274 \mathrm{~Hz}$ ), 121.4 (q apparent d, $J_{C F}=30 \mathrm{~Hz}$ ), $119.7\left(\mathrm{q}, J_{C F}=6 \mathrm{~Hz}\right), 118.8\left(\mathrm{q}, J_{C F}=321 \mathrm{~Hz}\right), 24.8$

HRMS Calculated for $\mathrm{C}_{10} \mathrm{H}_{7} \mathrm{~F}_{6} \mathrm{NO}_{4} \mathrm{~S}[\mathrm{M}+\mathrm{H}]^{+} 352.0073$, found 352.0067<smiles>CC(=O)Nc1ccc(O)cc1C</smiles>

\section{4-Acetamido-3-acetylphenyl trifluoromethanesulfonate $\mathbf{( 2 g )}$}

Prepared according to general procedure $\mathrm{C}\left(20^{\circ} \mathrm{C}\right)$, from acetanilide $1 \mathrm{~g}(55 \%$, white powder).

$\mathrm{mp} 83-84^{\circ} \mathrm{C}$

$\mathbf{R}_{\mathfrak{f}} 0.6$ (petroleum ether/EtOAc 70:30)

${ }^{1} \mathrm{H}$ NMR (400 MHz, $\left.\mathrm{CDCl}_{3}, 298 \mathrm{~K}\right) \delta 11.66(\mathrm{br} \mathrm{s}, \mathrm{NH}), 8.90(\mathrm{~d}, J=9.4 \mathrm{~Hz}, 1 \mathrm{H}), 7.76(\mathrm{~d}, J=2.9 \mathrm{~Hz}, 1 \mathrm{H}), 7.44(\mathrm{dd}, J=9.4$ and $2.9 \mathrm{~Hz}, 1 \mathrm{H}), 2.69(\mathrm{~s}, 3 \mathrm{H}), 2.25(\mathrm{~s}, 3 \mathrm{H})$

${ }^{19} \mathrm{~F}$ NMR (376 $\left.\mathrm{MHz}, \mathrm{CDCl}_{3}, 298 \mathrm{~K}\right) \delta-72.5$

${ }^{13} \mathrm{C}$ NMR (63 MHz, $\left.\mathrm{CDCl}_{3}, 298 \mathrm{~K}\right) \delta 201.3,169.7,143.2,141.0,127.7,124.2,122.6,122.4,118.8\left(\mathrm{q}, J_{C F}=321 \mathrm{~Hz}\right)$, 28.7, 25.6

HRMS Calculated for $\mathrm{C}_{11} \mathrm{H}_{10} \mathrm{~F}_{3} \mathrm{NO}_{5} \mathrm{~S}[\mathrm{M}+\mathrm{H}]^{+} 326.0305$, found 326.0304 
<smiles>CC(=O)Nc1ccc(O)cc1C</smiles>

4-Acetamido-3-methylphenyl trifluoromethanesulfonate (2h)

Prepared according to general procedure $\mathrm{C}\left(20^{\circ} \mathrm{C}\right)$, from acetanilide $1 \mathrm{~h}(28 \%$, white powder).

mp $98-99^{\circ} \mathrm{C}$

$\mathbf{R}_{\mathbf{f}} 0.1$ (petroleum ether/EtOAc 70:30)

${ }^{1} \mathrm{H}$ NMR (400 MHz, $\left.\mathrm{CDCl}_{3}, 298 \mathrm{~K}\right) \delta 7.94(\mathrm{~d}, J=9.7 \mathrm{~Hz}, 1 \mathrm{H}), 7.12-7.09(\mathrm{~m}, 2 \mathrm{H}), 7.07(\mathrm{br} \mathrm{s}, \mathrm{NH}), 2.28(\mathrm{~s}, 3 \mathrm{H}), 2.22(\mathrm{~s}$, 3H)

${ }^{19} \mathrm{~F}$ NMR (376 $\left.\mathrm{MHz}, \mathrm{CDCl}_{3}, 298 \mathrm{~K}\right) \delta-72.8$

${ }^{13} \mathrm{C}$ NMR (100 MHz, $\left.\mathrm{CDCl}_{3}, 298 \mathrm{~K}\right) \delta 168.4,145.8,135.8,131.1,124.3,123.1,119.5,118.7$ (q, $\left.J_{C F}=321 \mathrm{~Hz}\right), 24.4,17.9$ HRMS Calculated for $\mathrm{C}_{10} \mathrm{H}_{10} \mathrm{~F}_{3} \mathrm{NO}_{4} \mathrm{~S}[\mathrm{M}+\mathrm{H}]^{+} 298.0355$, found 298.0348<smiles>CC(=O)Nc1ccc(O)c(I)c1</smiles>

4-Acetamido-2-iodophenyl trifluoromethanesulfonate (2i)

Prepared according to general procedure $\mathrm{C}\left(50^{\circ} \mathrm{C}\right)$, from acetanilide $1 \mathbf{i}(42 \%$, colorless oil).

$\mathbf{R}_{\mathrm{f}} 0.3$ (petroleum ether/EtOAc 70:30)

${ }^{1} \mathrm{H}-\mathrm{NMR}\left(400 \mathrm{MHz}, \mathrm{CDCl}_{3}, 298 \mathrm{~K}\right) \delta 8.11(\mathrm{~d}, J=2.6 \mathrm{~Hz}, 1 \mathrm{H}), 7.58(\mathrm{dd}, J=9.0$ and $2.6 \mathrm{~Hz}, 1 \mathrm{H}), 7.29(\mathrm{br} \mathrm{s}, \mathrm{NH}), 7.24(\mathrm{~d}$, $J=9.0 \mathrm{~Hz}, 1 \mathrm{H}), 2.19(\mathrm{~s}, 3 \mathrm{H})$

${ }^{19} \mathrm{~F}$ NMR (376 MHz, $\left.\mathrm{CDCl}_{3}, 298 \mathrm{~K}\right) \delta-73.1$

${ }^{13} \mathrm{C}$ NMR (100 MHz, $\left.\mathrm{CDCl}_{3}, 298 \mathrm{~K}\right) \delta 168.7,146.3,138.6,131.0,122.2,120.8,118.8\left(\mathrm{q}, J_{C F}=321 \mathrm{~Hz}\right), 89.5,24.7$

HRMS Calculated for $\mathrm{C}_{9} \mathrm{H}_{7} \mathrm{~F}_{3} \mathrm{INO}_{4} \mathrm{~S}[\mathrm{M}+\mathrm{H}]^{+} 409.9165$, found 409.9157<smiles>CC(=O)Nc1ccc(O)c(Br)c1</smiles>

4-Acetamido-2-bromophenyl trifluoromethanesulfonate (2j)

Prepared according to general procedure $\mathrm{C}\left(50^{\circ} \mathrm{C}\right)$, from acetanilide $1 \mathbf{j}(50 \%$, brown oil).

$\mathbf{R}_{\mathrm{f}} 0.2$ (petroleum ether/EtOAc 70:30)

${ }^{1} \mathrm{H}$ NMR $\left(400 \mathrm{MHz}, \mathrm{CDCl}_{3}, 298 \mathrm{~K}\right) \delta 7.98(\mathrm{~d}, J=2.6 \mathrm{~Hz}, 1 \mathrm{H}), 7.50(\mathrm{dd}, J=9.0$ and $2.6 \mathrm{~Hz}, 1 \mathrm{H}), 7.31(\mathrm{br} \mathrm{s}, \mathrm{NH}), 7.28(\mathrm{~d}$, $J=9.1 \mathrm{~Hz}, 1 \mathrm{H}), 2.20(\mathrm{~s}, 3 \mathrm{H})$

${ }^{19} \mathrm{~F}$ NMR (376 MHz, $\left.\mathrm{CDCl}_{3}, 298 \mathrm{~K}\right) \delta-73.3$

${ }^{13} \mathrm{C}$ NMR (100 MHz, $\left.\mathrm{CDCl}_{3}, 298 \mathrm{~K}\right) \delta 168.5,138.6,124.8,123.2,119.6,118.8$ (q apparent $\mathrm{d}, J_{C F}=321 \mathrm{~Hz}$ ), 116.5, 108.5, 24.8

HRMS Calculated for $\mathrm{C}_{9} \mathrm{H}_{7} \mathrm{BrF}_{3} \mathrm{NO}_{4} \mathrm{~S}[\mathrm{M}+\mathrm{H}]^{+} 361.9304$, found 361.9305 
<smiles>CC(=O)Nc1ccc(O)c(Cl)c1</smiles>

\section{4-Acetamido-2-chlorophenyl trifluoromethanesulfonate (2k)}

Prepared according to general procedure $\mathrm{C}\left(50^{\circ} \mathrm{C}\right)$, from acetanilide $1 \mathbf{k}(62 \%$, yellow oil).

$\mathbf{R}_{\mathrm{f}} 0.2$ (petroleum ether/EtOAc $70: 30$ )

${ }^{1} \mathrm{H}$ NMR (400 MHz, $\left.\mathrm{CDCl}_{3}, 298 \mathrm{~K}\right) \delta 8.00(\mathrm{br} \mathrm{s}, \mathrm{NH}), 7.85(\mathrm{~d}, J=2.6 \mathrm{~Hz}, 1 \mathrm{H}), 7.41(\mathrm{dd}, J=9.0$ and $2.6 \mathrm{~Hz}, 1 \mathrm{H}), 7.25(\mathrm{~d}$, $J=9.3 \mathrm{~Hz}, 1 \mathrm{H}), 2.19(\mathrm{~s}, 3 \mathrm{H})$

${ }^{19} \mathrm{~F}$ NMR (376 $\left.\mathrm{MHz}, \mathrm{CDCl}_{3}, 298 \mathrm{~K}\right) \delta-73.4$

${ }^{13} \mathrm{C}$ NMR (100 MHz, $\left.\mathrm{CDCl}_{3}, 298 \mathrm{~K}\right) \delta 169.1,141.5,138.7,127.8,123.3,121.9,119.1,118.7\left(\mathrm{q}, J_{C F}=321 \mathrm{~Hz}\right), 24.6$

HRMS Calculated for $\mathrm{C}_{9} \mathrm{H}_{7} \mathrm{ClF}_{3} \mathrm{NO}_{4} \mathrm{~S}[\mathrm{M}+\mathrm{H}]^{+} 317.9809$, found 317.9815<smiles>CC(=O)Nc1ccc(O)c(F)c1</smiles>

\section{4-Acetamido-2-fluorophenyl trifluoromethanesulfonate (2I)}

Prepared according to general procedure $\mathrm{C}\left(50^{\circ} \mathrm{C}\right)$, from acetanilide 1 (I (58\%, white powder).

mp $83-84^{\circ} \mathrm{C}$

$\mathbf{R}_{\mathrm{f}} 0.15$ (petroleum ether/EtOAc 70:30)

${ }^{1} \mathrm{H}$ NMR (400 MHz, $\left.\mathrm{CDCl}_{3}, 298 \mathrm{~K}\right) \delta 7.81(\mathrm{br} \mathrm{s}, \mathrm{NH}), 7.76(\mathrm{dd}, J=11.9$ and $2.4 \mathrm{~Hz}, 1 \mathrm{H}), 7.24(\mathrm{dd}, J=7.9$ and $7.9 \mathrm{~Hz}$, $1 \mathrm{H}), 7.17(\mathrm{ddd}, J=9.0,2.4$ and $1.3 \mathrm{~Hz}, 1 \mathrm{H}), 2.19(\mathrm{~s}, 3 \mathrm{H})$

${ }^{19} \mathrm{~F}$ NMR (376 $\left.\mathrm{MHz}, \mathrm{CDCl}_{3}, 298 \mathrm{~K}\right) \delta-73.15(3 \mathrm{~F}),-125.0(1 \mathrm{~F})$

$\left.{ }^{13} \mathrm{C} \mathrm{NMR} \mathrm{(63} \mathrm{MHz,} \mathrm{CDCl}_{3}, 298 \mathrm{~K}\right) \delta 168.9,153.8\left(\mathrm{~d}, J_{C F}=252 \mathrm{~Hz}\right), 139.2\left(\mathrm{~d}, J_{C F}=10 \mathrm{~Hz}\right), 132.5\left(\mathrm{~d}, J_{C F}=14 \mathrm{~Hz}\right), 123.7$, $118.8(\mathrm{q}, J=321 \mathrm{~Hz}), 115.3\left(\mathrm{~d}, J_{C F}=3 \mathrm{~Hz}\right), 109.1\left(\mathrm{~d}, J_{C F}=23 \mathrm{~Hz}\right), 24.7$

HRMS Calculated for $\mathrm{C}_{9} \mathrm{H}_{7} \mathrm{~F}_{4} \mathrm{NO}_{4} \mathrm{~S}[\mathrm{M}+\mathrm{H}]^{+} 302.0105$, found 302.0113<smiles>CC(=O)Nc1ccc(O)c(C)c1</smiles>

\section{4-Acetamido-2-acetylphenyl trifluoromethanesulfonate $(2 \mathrm{~m})$}

Prepared according to general procedure $\mathrm{C}\left(50^{\circ} \mathrm{C}\right)$, from acetanilide $1 \mathrm{~m}(62 \%$, white powder).

mp $111-113^{\circ} \mathrm{C}$

$\mathbf{R}_{\mathrm{f}} 0.1$ (petroleum ether/EtOAc 70:30)

${ }^{1} \mathrm{H}$ NMR $\left(400 \mathrm{MHz}, \mathrm{CDCl}_{3}, 298 \mathrm{~K}\right) \delta 8.01(\mathrm{~d}, J=2.7 \mathrm{~Hz}, 1 \mathrm{H}), 7.77(\mathrm{dd}, J=8.9$ and $2.8 \mathrm{~Hz}, 1 \mathrm{H}), 7.54(\mathrm{br} s, \mathrm{NH}), 7.27(\mathrm{~d}$, $J=9.1 \mathrm{~Hz}, 2 \mathrm{H}), 2.63(\mathrm{~s}, 3 \mathrm{H}), 2.22(\mathrm{~s}, 3 \mathrm{H})$

${ }^{19} \mathrm{~F}$ NMR (376 $\left.\mathrm{MHz}, \mathrm{CDCl}_{3}, 298 \mathrm{~K}\right) \delta-73.2$

${ }^{13} \mathrm{C}$ NMR (100 MHz, $\left.\mathrm{CDCl}_{3}, 298 \mathrm{~K}\right) \delta 196.5,168.6,142.2,138.1,132.5,123.9,123.4,121.2,118.6\left(\mathrm{q}, \mathrm{J}_{C F}=321 \mathrm{~Hz}\right)$, 29.5, 24.6

HRMS Calculated for $\mathrm{C}_{11} \mathrm{H}_{10} \mathrm{~F}_{3} \mathrm{NO}_{5} \mathrm{~S}[\mathrm{M}+\mathrm{H}]^{+} 326.0305$, found 326.0319 
<smiles>COc1cccc(O)c1NC(C)=O</smiles>

Methyl 5-acetamido-2-(((trifluoromethyl)sulfonyl)oxy)benzoate (2n)

Prepared according to general procedure $\mathrm{C}\left(50^{\circ} \mathrm{C}\right)$, from acetanilide $1 \mathrm{n}(71 \%$, grey powder).

mp $150-152{ }^{\circ} \mathrm{C}$

$\mathbf{R}_{\mathbf{f}} 0.1$ (petroleum ether/EtOAc 70:30)

${ }^{1} \mathrm{H}$ NMR $\left(400 \mathrm{MHz}, \mathrm{CDCl}_{3}, 298 \mathrm{~K}\right) \delta 8.05(\mathrm{dd}, J=8.9$ and $2.8 \mathrm{~Hz}, 1 \mathrm{H}), 8.00(\mathrm{~d}, J=2.8 \mathrm{~Hz}, 1 \mathrm{H}), 7.51(\mathrm{~s}, 1 \mathrm{H}), 7.25(\mathrm{~d}$, $J=8.9 \mathrm{~Hz}, 1 \mathrm{H}), 3.96(\mathrm{~s}, 3 \mathrm{H}), 2.22(\mathrm{~s}, 3 \mathrm{H})$

$\left.{ }^{19} \mathrm{~F} \mathrm{NMR} \mathrm{(376} \mathrm{MHz}, \mathrm{CDCl}_{3}, 298 \mathrm{~K}\right) \delta-73.4$

${ }^{13} \mathrm{C}$ NMR (100 MHz, $\left.\mathrm{CDCl}_{3}, 298 \mathrm{~K}\right) \delta 168.7,164.0,143.9,138.1,124.99,124.88,123.7,123.0,118.8$ (q apparent d, $\left.J_{C F}=321 \mathrm{~Hz}\right), 53.0,24.7$

HRMS Calculated for $\mathrm{C}_{11} \mathrm{H}_{10} \mathrm{~F}_{3} \mathrm{NO}_{6} \mathrm{~S}[\mathrm{M}+\mathrm{H}]^{+} 342.0254$, found 342.0261<smiles>CC(=O)Nc1ccc(O)c(S(C)(=O)=O)c1</smiles>

4-Acetamido-2-(trimethylsilyl)phenyl trifluoromethanesulfonate (20)

Prepared according to general procedure $\mathrm{C}\left(20^{\circ} \mathrm{C}\right)$, from acetanilide $10(41 \%$, grey powder).

mp $128-130^{\circ} \mathrm{C}$

$\mathbf{R}_{\mathbf{f}} 0.2$ (petroleum ether/EtOAc 70:30)

${ }^{1} \mathrm{H}$ NMR $\left(400 \mathrm{MHz}, \mathrm{CDCl}_{3}, 298 \mathrm{~K}\right) \delta 7.72(\mathrm{dd}, J=9.0$ and $2.8 \mathrm{~Hz}, 1 \mathrm{H}), 7.50(\mathrm{~d}, J=2.7 \mathrm{~Hz}, 1 \mathrm{H}), 7.28(\mathrm{~d}, J=9.0 \mathrm{~Hz}, 1 \mathrm{H})$, $2.20(\mathrm{~s}, 3 \mathrm{H}), 0.36(\mathrm{~s}, 9 \mathrm{H})$

${ }^{19} \mathrm{~F}$ NMR (376 $\left.\mathrm{MHz}, \mathrm{CDCl}_{3}, 298 \mathrm{~K}\right) \delta-73.9$

${ }^{13} \mathrm{C}$ NMR (100 MHz, $\left.\mathrm{CDCl}_{3}, 298 \mathrm{~K}\right) \delta 168.3,150.6,136.9,133.7,126.7,122.3,120.3,118.6\left(\mathrm{q}, J_{C F}=320 \mathrm{~Hz}\right), 24.6,-0.9$ HRMS Calculated for $\mathrm{C}_{12} \mathrm{H}_{16} \mathrm{~F}_{3} \mathrm{NO}_{4} \mathrm{SSi}[\mathrm{M}+\mathrm{H}]^{+} 356.0594$, found 356.0600<smiles>CC(=O)Nc1ccc(O)c(C)c1</smiles>

4-Acetamido-2-methylphenyl trifluoromethanesulfonate (2p)

Prepared according to general procedure $\mathrm{C}\left(20^{\circ} \mathrm{C}\right)$, from acetanilide $1 \mathrm{p}(26 \%$, white powder).

$\mathrm{mp} 72-73^{\circ} \mathrm{C}$

$\mathbf{R}_{\mathbf{f}} 0.2$ (petroleum ether/EtOAc 50:50)

${ }^{1} \mathrm{H}$ NMR (400 MHz, $\left.\mathrm{CDCl}_{3}, 298 \mathrm{~K}\right) \delta 7.53(\mathrm{~d}, J=2.3 \mathrm{~Hz}, 1 \mathrm{H}), 7.36(\mathrm{dd}, J=8.9$ and $2.5 \mathrm{~Hz}, 1 \mathrm{H}), 7.32(\mathrm{br} \mathrm{s}, \mathrm{NH}), 7.17(\mathrm{~d}$, $J=8.9 \mathrm{~Hz}, 1 \mathrm{H}), 2.35(\mathrm{~s}, 3 \mathrm{H}), 2.18(\mathrm{~s}, 3 \mathrm{H})$

${ }^{19} \mathrm{~F}$ NMR (376 $\left.\mathrm{MHz}, \mathrm{CDCl}_{3}, 298 \mathrm{~K}\right) \delta-73.7$

${ }^{13} \mathrm{C}$ NMR $\left(100 \mathrm{MHz}, \mathrm{CDCl}_{3}, 298 \mathrm{~K}\right.$ ) $\delta 168.7,144.5,137.6,132.0,123.0,122.0,118.7,118.7$ (q apparent d, $J_{C F}=321$ $\mathrm{Hz}), 24.7,16.7$

HRMS Calculated for $\mathrm{C}_{10} \mathrm{H}_{10} \mathrm{~F}_{3} \mathrm{NO}_{4} \mathrm{~S}[\mathrm{M}+\mathrm{H}]^{+} 298.0355$, found 298.0362 
<smiles>CC(=O)Nc1ccc(O)c(OC(C)=O)c1</smiles>

5-Acetamido-2-(((trifluoromethyl)sulfonyl)oxy)phenyl acetate (2r)

Prepared according to general procedure $\mathrm{C}\left(20^{\circ} \mathrm{C}\right)$, from acetanilide $1 \mathrm{r}(64 \%$, white powder $)$.

mp $109-111^{\circ} \mathrm{C}$

$\mathbf{R}_{\mathbf{f}} 0.1$ (petroleum ether/EtOAc 70:30)

${ }^{1} \mathrm{H}$ NMR $\left(400 \mathrm{MHz}, \mathrm{CDCl}_{3}, 298 \mathrm{~K}\right) \delta 7.77(\mathrm{~d}, J=2.5 \mathrm{~Hz}, 1 \mathrm{H}), 7.61(\mathrm{br} \mathrm{s}, \mathrm{NH}), 7.16(\mathrm{~d}, J=9.0 \mathrm{~Hz}, 1 \mathrm{H}), 7.11$ (dd, $J=9.0$ and $2.5 \mathrm{~Hz}, 1 \mathrm{H}), 2.37(\mathrm{~s}, 3 \mathrm{H}), 2.15(\mathrm{~s}, 3 \mathrm{H})$

$\left.{ }^{19} \mathrm{~F} \mathrm{NMR} \mathrm{(376} \mathrm{MHz,} \mathrm{CDCl}_{3}, 298 \mathrm{~K}\right) \delta-73.7$

${ }^{13} \mathrm{C}$ NMR (100 MHz, $\left.\mathbf{C D C l}_{3}, 298 \mathrm{~K}\right) \delta 168.8,168.5,142.5,138.6,136.5,122.8,118.7$ (q apparent d, $J_{C F}=321 \mathrm{~Hz}$ ), $117.5,115.5,24.7,20.8$

HRMS Calculated for $\mathrm{C}_{11} \mathrm{H}_{10} \mathrm{~F}_{3} \mathrm{NO}_{6} \mathrm{~S}[\mathrm{M}+\mathrm{H}]^{+} 342.0254$, found 342.0266 


\section{Synthesis and Characterization of Arylnonaflates 3a, 3e, 3l and 3r}<smiles>COc1ccc(NC(C)=O)cc1</smiles>

\section{4-Acetamidophenyl nonafluorobutanesulfonate (3a)}

Prepared according to general procedure $\mathrm{C}\left(20^{\circ} \mathrm{C}\right)$, from acetanilide $1 \mathrm{a}(58 \%$, brown powder).

mp $110-112^{\circ} \mathrm{C}$

$\mathbf{R}_{\mathfrak{f}} 0.25$ (petroleum ether/EtOAc 70:30)

${ }^{1} \mathrm{H}$ NMR (400 MHz, $\left.\mathrm{CDCl}_{3}, 298 \mathrm{~K}\right) \delta 7.60(\mathrm{~d}, J=9.1 \mathrm{~Hz}, 2 \mathrm{H}), 7.30(\mathrm{br} \mathrm{s}, \mathrm{NH}), 7.24(\mathrm{~d}, J=9.1 \mathrm{~Hz}, 2 \mathrm{H}), 2.20(\mathrm{~s}, 3 \mathrm{H})$

${ }^{19} \mathrm{~F}$ NMR $\left(376 \mathrm{MHz}, \mathrm{CDCl}_{3}, 298 \mathrm{~K}\right) \delta-80.6(\mathrm{t}, J=10 \mathrm{~Hz}, 3 \mathrm{~F}),-108.9(\mathrm{t}, J=14 \mathrm{~Hz}, 2 \mathrm{~F}),-120.9(\mathrm{~m}, 2 \mathrm{~F}),-125.8(\mathrm{~m}, 2 \mathrm{~F})$

${ }^{13} \mathrm{C}$ NMR (100 MHz, $\left.\mathbf{C D C l}_{3}, 298 \mathrm{~K}\right) \delta$ 168.3, 145.5, 137.8, 122.0, 120.9, 24.6

HRMS Calculated for $\mathrm{C}_{12} \mathrm{H}_{8} \mathrm{~F}_{9} \mathrm{NO}_{4} \mathrm{~S}[\mathrm{M}+\mathrm{H}]^{+} 434.0103$, found 434.0124<smiles>CC(=O)Nc1ccc(O)cc1Cl</smiles>

4-Acetamido-3-chlorophenyl nonafluorobutanesulfonate (3e)

Prepared according to general procedure $\mathrm{C}\left(20^{\circ} \mathrm{C}\right)$, from acetanilide $1 \mathrm{e}(65 \%$, light brown powder).

$\mathrm{mp} 85-87^{\circ} \mathrm{C}$

$\mathbf{R}_{\mathfrak{f}} 0.4$ (petroleum ether/EtOAc 70:30)

${ }^{1} \mathrm{H}$ NMR $\left(400 \mathrm{MHz}, \mathrm{CDCl}_{3}, 298 \mathrm{~K}\right) \delta 8.55(\mathrm{~d}, J=9.2 \mathrm{~Hz}, 1 \mathrm{H}), 7.66(\mathrm{br} \mathrm{s}, \mathrm{NH}), 7.37(\mathrm{~d}, J=2.8 \mathrm{~Hz}, 1 \mathrm{H}), 7.23(\mathrm{dd}, J=9.2$ and $2.8 \mathrm{~Hz}, 1 \mathrm{H}), 2.29(\mathrm{~s}, 3 \mathrm{H})$

${ }^{19} \mathrm{~F}$ NMR (376 MHz, $\left.\mathrm{CDCl}_{3}, 298 \mathrm{~K}\right) \delta-80.6(\mathrm{t}, J=10 \mathrm{~Hz}, 3 \mathrm{~F}),-108.7(\mathrm{t}, J=14 \mathrm{~Hz}, 2 \mathrm{~F}),-120.9(\mathrm{~m}, 2 \mathrm{~F}),-125.8(\mathrm{~m}, 2 \mathrm{~F})$

${ }^{13} \mathrm{C}$ NMR (100 MHz, $\left.\mathrm{CDCl}_{3}, 298 \mathrm{~K}\right) \delta$ 168.3, 144.5, 134.9, 122.9, 122.2, 122.1, 120.8, 24.9

HRMS Calculated for $\mathrm{C}_{12} \mathrm{H}_{7} \mathrm{ClF}_{9} \mathrm{NO}_{4} \mathrm{~S}[\mathrm{M}+\mathrm{H}]^{+} 467.9713$, found 467.9702<smiles>CC(=O)Nc1ccc(O)c(F)c1</smiles>

N-(3-Fluoro-4-(perfluorobutoxy)phenyl)acetamide (3l)

Prepared according to general procedure $\mathrm{C}\left(20^{\circ} \mathrm{C}\right)$, from acetanilide $1 \mathrm{I}(65 \%$, yellow powder).

mp $79-80^{\circ} \mathrm{C}$

$\mathbf{R}_{\mathbf{f}} 0.15$ (petroleum ether/EtOAc 70:30)

${ }^{1} \mathrm{H}$ NMR (400 MHz, MeOD, 298 K) $\delta 7.86(\mathrm{dd}, J=12.7,2.3 \mathrm{~Hz}, 1 \mathrm{H}), 7.41-7.32(\mathrm{~m}, 2 \mathrm{H}), 2.14(\mathrm{~s}, 3 \mathrm{H})$

${ }^{19} \mathrm{~F}$ NMR (376 MHz, MeOD, $\left.298 \mathrm{~K}\right) \delta-82.3(\mathrm{t}, J=10 \mathrm{~Hz}, 3 \mathrm{~F}),-110.8(\mathrm{t}, J=14 \mathrm{~Hz}, 2 \mathrm{~F}),-122.0(\mathrm{~m}, 2 \mathrm{~F}),-127.1(\mathrm{~m}$, $2 \mathrm{~F}),-128.0(\mathrm{~s}, 1 \mathrm{~F})$

${ }^{13} \mathrm{C}$ NMR (100 MHz, MeOD, $\left.298 \mathrm{~K}\right) \delta 171.9,154.8$ (d, $\left.J_{C F}=249 \mathrm{~Hz}, 1 \mathrm{C}\right), 141.8$ (d, $\left.J_{C F}=10 \mathrm{~Hz}, 1 \mathrm{C}\right), 133.4$ (d, $\left.J_{C F}=14 \mathrm{~Hz}, 1 \mathrm{C}\right), 124.7,116.7\left(\mathrm{~d}, J_{C F}=3 \mathrm{~Hz}, 1 \mathrm{C}\right), 109.3\left(\mathrm{~d}, J_{C F}=24 \mathrm{~Hz}, 1 \mathrm{C}\right), 23.9$

HRMS Calculated for $\mathrm{C}_{12} \mathrm{H}_{7} \mathrm{~F}_{10} \mathrm{NO}_{4} \mathrm{~S}[\mathrm{M}+\mathrm{H}]^{+} 452.0009$, found 452.0020 


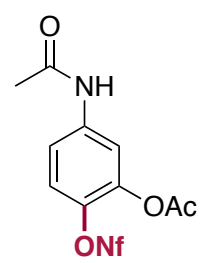

5-Acetamido-2-(perfluorobutoxy)phenyl acetate (3r)

Prepared according to general procedure $\mathrm{C}\left(20^{\circ} \mathrm{C}\right)$, from acetanilide $1 \mathrm{r}(42 \%$, yellow powder).

mp $107-108^{\circ} \mathrm{C}$

$\mathbf{R}_{\mathfrak{f}} 0.1$ (petroleum ether/EtOAc 70:30)

${ }^{1} \mathrm{H}$ NMR $\left(400 \mathrm{MHz}, \mathrm{CDCl}_{3}, 298 \mathrm{~K}\right) \delta 7.79(\mathrm{~d}, J=2.5 \mathrm{~Hz}, 1 \mathrm{H}), 7.64(\mathrm{~s}, 1 \mathrm{H}), 7.13(\mathrm{~d}, J=9.0 \mathrm{~Hz}, 1 \mathrm{H}), 7.06(\mathrm{dd}, J=9.0$ and $2.6 \mathrm{~Hz}, 1 \mathrm{H}), 2.37(\mathrm{~s}, 3 \mathrm{H}), 2.15(\mathrm{~s}, 3 \mathrm{H})$

${ }^{19} \mathrm{~F}$ NMR $\left(376 \mathrm{MHz}, \mathrm{CDCl}_{3}, 298 \mathrm{~K}\right) \delta-80.6(\mathrm{t}, J=10 \mathrm{~Hz}, 3 \mathrm{~F}),-109.6(\mathrm{t}, J=14 \mathrm{~Hz}, 2 \mathrm{~F}),-120.8(\mathrm{~m}, 2 \mathrm{~F}),-125.9(\mathrm{~m}, 2 \mathrm{~F})$

${ }^{13} \mathrm{C}$ NMR $\left(100 \mathrm{MHz}, \mathrm{CDCl}_{3}, 298 \mathrm{~K}\right) \delta 168.8,168.7,142.5,138.7,136.5,122.6,117.4,115.4,24.7,20.8$

HRMS Calculated for $\mathrm{C}_{14} \mathrm{H}_{10} \mathrm{~F}_{9} \mathrm{NO}_{6} \mathrm{~S}[\mathrm{M}+\mathrm{H}]^{+} 492.0158$, found 492.0151 


\section{Synthesis and Characterization of Aryltriflates 5a-c}<smiles>CC(C)(C)C(=O)Nc1ccc(Oc2ccccc2)cc1</smiles>

\section{4-Pivalamidophenyl trifluoromethanesulfonate (5a)}

Prepared according to general procedure $\mathrm{C}\left(20^{\circ} \mathrm{C}\right)$, from $\mathrm{N}$-pivaloylaniline $4 \mathrm{a}(50 \%$, white powder).

mp $130-132{ }^{\circ} \mathrm{C}$

$\mathbf{R}_{\mathfrak{f}} 0.3$ (petroleum ether/EtOAc 90:10)

${ }^{1} \mathrm{H}$ NMR (400 MHz, $\left.\mathrm{CDCl}_{3}, 298 \mathrm{~K}\right) \delta 7.63(\mathrm{~d}, J=9.1 \mathrm{~Hz}, 2 \mathrm{H}), 7.43(\mathrm{br} \mathrm{s}, \mathrm{NH}), 7.23(\mathrm{~d}, J=8.8 \mathrm{~Hz}, 2 \mathrm{H}), 1.32(\mathrm{~s}, 9 \mathrm{H})$

${ }^{19} \mathrm{~F}$ NMR (376 MHz, $\left.\mathrm{CDCl}_{3}, 298 \mathrm{~K}\right) \delta-72.7$

${ }^{13} \mathrm{C}$ NMR $\left(100 \mathrm{MHz}, \mathrm{CDCl}_{3}, 298 \mathrm{~K}\right) \delta 177.0,145.3,138.2,122.0,121.4,118.8$ (q apparent d, $\left.J_{C F}=321 \mathrm{~Hz}\right), 39.8,27.6$

HRMS Calculated for $\mathrm{C}_{12} \mathrm{H}_{14} \mathrm{~F}_{3} \mathrm{NO}_{4} \mathrm{~S}[\mathrm{M}+\mathrm{H}]^{+} 326.0668$, found 326.0664<smiles>O=C(Nc1ccc(Br)cc1)c1ccccc1</smiles>

\section{4-Benzamidophenyl trifluoromethanesulfonate (5b)}

Prepared according to general procedure $\mathrm{C}\left(20^{\circ} \mathrm{C}\right)$, from $\mathrm{N}$-benzoylaniline $4 \mathrm{~b}$ ( $30 \%$, light pink powder).

mp $180-182{ }^{\circ} \mathrm{C}$

$\mathbf{R}_{\mathrm{f}} 0.2$ (petroleum ether/EtOAc $90: 10$ )

${ }^{1} \mathrm{H}$ NMR (400 MHz, $\left.\mathrm{CDCl}_{3}, 298 \mathrm{~K}\right) \delta 7.92(\mathrm{br} \mathrm{s}, \mathrm{NH}), 7.87(\mathrm{dd}, J=8.3$ and 1.3 Hz, 2H), 7.77-7.74 (m, 2H), $7.59(\mathrm{~m}, 1 \mathrm{H})$, $7.51(\mathrm{tt}, J=7.4$ and $1.5 \mathrm{~Hz}, 2 \mathrm{H}), 7.30-7.28(\mathrm{~m}, 2 \mathrm{H})$

${ }^{19} \mathrm{~F}$ NMR (376 MHz, $\left.\mathrm{CDCl}_{3}, 298 \mathrm{~K}\right) \delta-72.7$

${ }^{13} \mathrm{C}$ NMR (100 MHz, $\left.\mathrm{CDCl}_{3}, 298 \mathrm{~K}\right) \delta 165.9,145.6,138.1,134.4,132.4,129.1,127.2,122.2,121.6$

HRMS Calculated for $\mathrm{C}_{14} \mathrm{H}_{10} \mathrm{~F}_{3} \mathrm{NO}_{4} \mathrm{~S}[\mathrm{M}+\mathrm{H}]^{+} 346.0355$, found 346.0356<smiles>O=C(Nc1ccc(Br)cc1)OCc1ccccc1</smiles>

4-(((Benzyloxy)carbonyl)amino)phenyl trifluoromethanesulfonate (5c)

Prepared according to modified general procedure $\mathrm{C}$ (using 1.5 equiv of $\mathrm{Phl}(\mathrm{OAc})_{2}$ and 2.0 equiv of $\mathrm{AgOTf}$ in $\mathrm{CHCl}_{3}$ at $\left.20^{\circ} \mathrm{C}\right)$, from benzyl phenylcarbamate $4 \mathrm{c}(60 \%$, light brown powder).

mp $95-97^{\circ} \mathrm{C}$

$\mathbf{R}_{\mathrm{f}} 0.4$ (petroleum ether/EtOAc 90:10)

${ }^{1} \mathrm{H}$ NMR (400 MHz, $\left.\mathrm{CDCl}_{3}, 298 \mathrm{~K}\right) \delta 7.49(\mathrm{~d}, J=8.9 \mathrm{~Hz}, 2 \mathrm{H}), 7.43-7.36(\mathrm{~m}, 5 \mathrm{H}), 7.24-7.22(\mathrm{~m}, 2 \mathrm{H}), 6.83(\mathrm{br} \mathrm{s}, \mathrm{NH}), 5.23$ $(\mathrm{s}, 2 \mathrm{H})$

${ }^{19} \mathrm{~F}$ NMR (376 $\left.\mathrm{MHz}, \mathrm{CDCl}_{3}, 298 \mathrm{~K}\right) \delta-72.7$

${ }^{13} \mathrm{C}$ NMR (100 MHz, $\left.\mathrm{CDCl}_{3}, 298 \mathrm{~K}\right) \delta 153.2,145.0,138.0,135.8,128.84,128.72,128.55,122.2,119.9,118.8$ (q apparent $\left.d, J_{C F}=321 \mathrm{~Hz}\right), 67.6$

HRMS Calculated for $\mathrm{C}_{15} \mathrm{H}_{12} \mathrm{~F}_{3} \mathrm{NO}_{5} \mathrm{~S}[\mathrm{M}+\mathrm{H}]^{+} 376.0461$, found 376.0449 


\section{Synthesis and Characterization of Diarylamide 6}<smiles>CN(c1ccccc1)c1ccc(I)cc1</smiles>

$N$-(4-lodophenyl)-N-phenylacetamide (6)

Prepared according to modified general procedure C (using HFIP instead of DCE, $20^{\circ} \mathrm{C}$ ), from acetanilide $1 \mathrm{a}(21 \%$, light brown powder).

Exhibited spectral data identical to a previous report. ${ }^{28}$

$\mathbf{R}_{\mathrm{f}} 0.4$ (petroleum ether/EtOAc 70:30)

${ }^{1} \mathrm{H}$ NMR (400 MHz, $\left.\mathrm{CDCl}_{3}, 298 \mathrm{~K}\right) \delta 7.65$ (d apparent br s, 2H), 7.50-7.28 (br m, 3H), $7.24(\mathrm{~d}, J=7.5 \mathrm{~Hz}, 2 \mathrm{H}), 7.02(\mathrm{~d}$, $J=8.8 \mathrm{~Hz}, 2 \mathrm{H}), 2.05(\mathrm{~s}, 3 \mathrm{H})$

HRMS Calculated for $\mathrm{C}_{14} \mathrm{H}_{12} \mathrm{INO}[\mathrm{M}+\mathrm{H}]^{+} 338.0036$, found 338.0013

${ }^{28}$ He, C.; Chen, C.; Cheng, J.; Liu, C.; Liu, W.; Li, Q.; Lei, A. Angew. Chem. Int. Ed. 2008, 47, 6414-6417. 


\section{Triflate Salt Solubilities (at $20^{\circ} \mathrm{C}$ )}

Procedure for the determination of the solubilities

$1 \mathrm{~mL}$ of a saturated solution of the triflate salt was filtered through a small piece of cotton in a pipette then evaporated and dried under reduced pressure.

\begin{tabular}{ccc}
\hline Triflate Salt & Solvent & Solubility $\left(\mathrm{mg}^{\mathrm{mL}}{ }^{-1}\right)$ \\
\hline $\mathrm{Cu}(\mathrm{OTf})_{2}$ & $\mathrm{CHCl}_{3}$ & 1.1 \\
$\mathrm{Zn}(\mathrm{OTf})_{2}$ & $\mathrm{CHCl}_{3}$ & 3.2 \\
$\mathrm{Fe}(\mathrm{OTf})_{2}$ & $\mathrm{CHCl}_{3}$ & 1.8 \\
$\mathrm{Ce}(\mathrm{OTf})_{3}$ & $\mathrm{DCE}$ & 2.4 \\
$(\mathrm{CuOTf})_{2} \cdot \mathrm{Tol}$ & $\mathrm{CHCl}_{3}$ & 2.1 \\
$\mathrm{NaOTf}$ & $\mathrm{DCE}$ & 0.9 \\
$\mathrm{KOTf}$ & $\mathrm{DCE}$ & 6.1 \\
$\mathrm{AgOTf}$ & $\mathrm{CHCl}$ & 4.4 \\
AgOTf & $\mathrm{DCE}$ & 3.1 \\
\hline
\end{tabular}

